\title{
Ileal Perforation Peritonitis Caused By T. Saginata: A Case Report
}

\author{
Kurane SB $^{1}$, Kurane BT $^{2}$ \\ ${ }^{1}$ Dr Sanjot B Kurane MS, ${ }^{2}$ Dr B T Kurane ${ }^{2}$ MS, both from Ganga Hospital, Miraj Maharashtra, India \\ Address for correspondence: Dr Sanjot B Kurane, Email: dr_sanjot@yahoo.co.in
}

\begin{abstract}
Worms are rare but definitive cause of intestinal perforation. Ascaris Lumbricoides is the main cause of intestinal perforation, especially in children. Other worms reported to cause perforation include Enterobius vermicularis, Trichuris trichura, and Tania solium. Tapeworm infestation is one of the common problems in tropical countries; usually they are asymptomatic but sometimes associated with serious complication like intestinal obstruction, appendicitis, or perforation. Few cases have been reported of perforation caused by T.solium in the literature, we present here a case report of tapeworm (T. Saginata) causing small bowel perforation and peritonitis.
\end{abstract}

Keywords: Ileal perforation, peritonitis, T. Saginata

\section{Introduction}

Perforation peritonitis is one of the most common surgical emergencies in India. Even with advances in peri-operative care, antimicrobial therapy and intensive care support, patients with peritonitis still suffer high morbidity and mortality ${ }^{1}$. Many patients present late with pre-established sepsis and septic shock, which are associated with high mortality. The spectrum of etiology continues to be different from that of western countries and there is paucity of data from India ${ }^{2}$

In India, duodenal ulcer perforation, appendicular perforation, enteric perforation, and tubercular perforation being a major cause for perforative peritonitis, as compared to western world where traumatic perforation accounts for maximum number of cases. Here we are presenting a case report of perforation with tapeworm peritonitis.

Manuscript received: $16^{\text {th }}$ July 2013

Reviewed: $26^{\text {th }}$ July 2013

Author Corrected: $29^{\text {th }}$ July 2013

Accepted for Publication: $30^{\text {th }}$ July 2013

\section{Case Report}

A 40 year old patient, Hindu by religion came with chief complaint of sudden onset severe pain in abdomen since one day. The pain was followed by 2-3 episodes of vomiting, Patients had tachycardia (pulse $=102$ beats $/ \mathrm{min}$ ). On per abdominal examination, patient had guarding and rigidity. In view of above findings clinical diagnosis of Perforative peritonitis was made.

His complete blood picture revealed hemoglobin$11.2 \mathrm{gm} \%$ and total leukocyte count- 11200 . His blood sugar level, HIV- test and renal functions were normal. Xray erect abdomen showed free gas under diaphragm. In view of above findings, diagnosis of perforative peritonitis was confirmed and patient was posted for emergency laparotomy. Intra operative- multiple flakes were present, and to our surprise there was ileal

perforation with tapeworm lying in peritoneal cavity (Fig1\& 2). Tapeworm removed and thorough warm saline peritoneal wash was given, perforation was closed primarily and omentoplasty done (Fig 3). 
Post operative period was uneventful. Patients was given Praziquantel $10-20 \mathrm{mg} / \mathrm{kg}$ body weight, he passed few more pieces of tapeworm. Histopathology report of worm

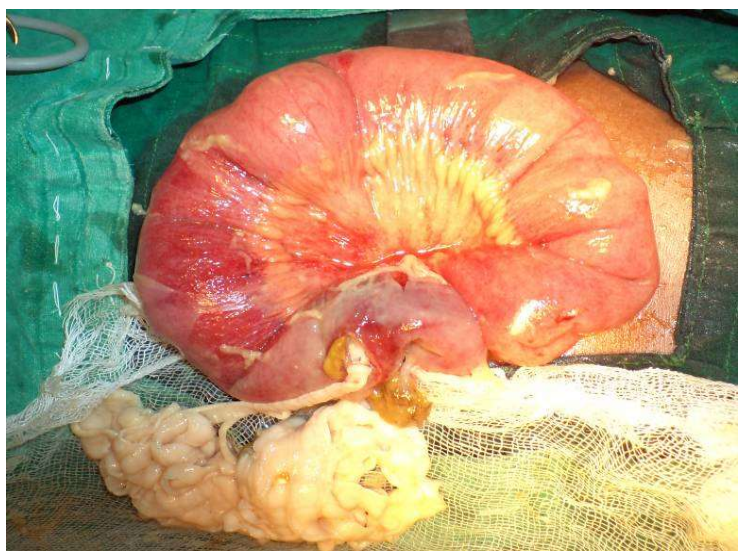

Figure 1- ileal perforation due to tapeworm

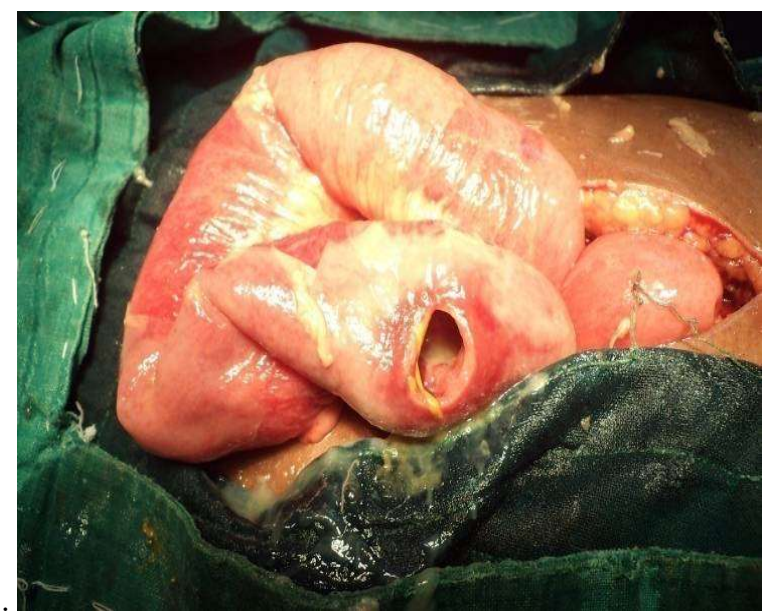

Figure 2: Perforation Site

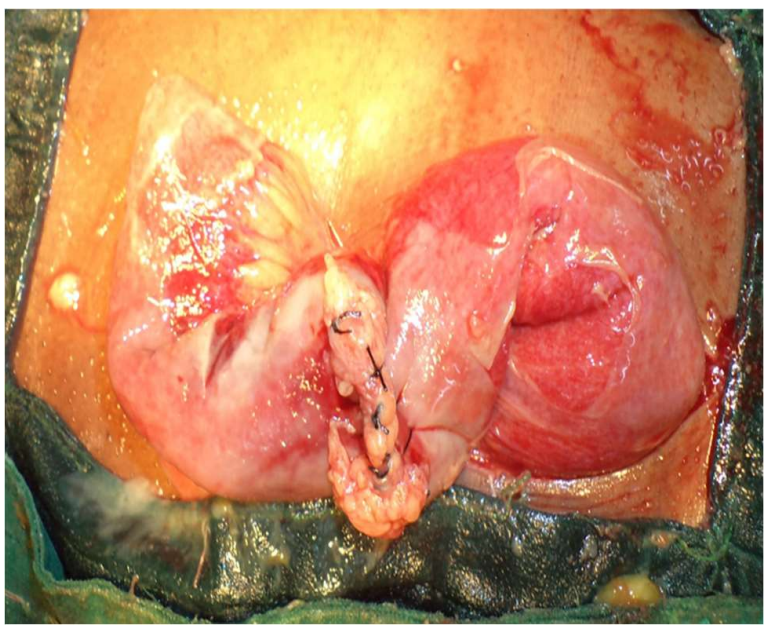

Figure 3- Closure of perforation confirmed it to be T. Saginata (Fig 4), and ulcer edge report was non-specific inflammation. WIDAL test was performed and it was negative.

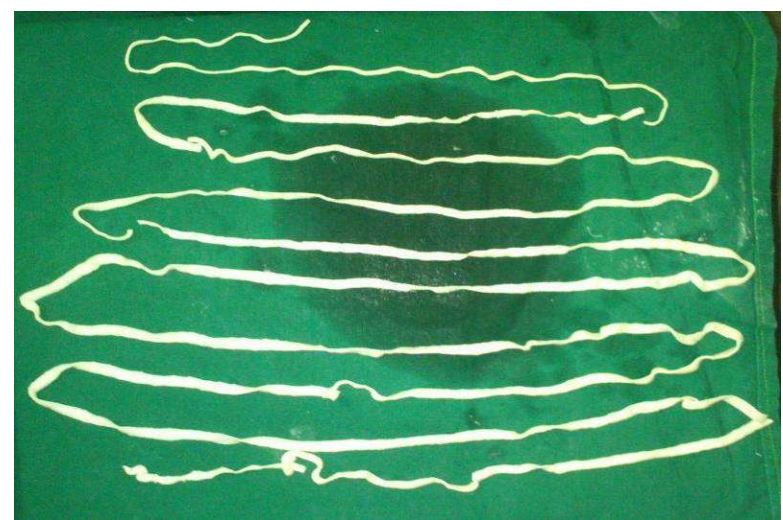

Figure 4- T. Saginata

\section{Discussion}

Taeniasis prevalence in tropical countries is high ${ }^{3}$. Tapeworm infestation is the infection of the digestive tract by adult tapeworms; larvae are sometimes ingested by consuming undercooked food.

Once inside the digestive tract larvae grow in to a very large adult tapeworm. Larvae can cause symptoms in as intermediate host, e.g. cysticercosis in humans.

Among the most common tapeworms in humans are the beef tapeworm ( $T$. saginata), the pork tape (T. solium), the fish tapeworm (Diphyllobothrium spp.), and the dwarf tapeworm (Hymenolepis spp.). Infections involving the pork and beef tapeworms are also called taeniasis

Taenia Saginata is the beef tapeworm. Man is the only definitive host, and cattle are the significant intermediate host, though a variety of ungulates have been reported as being infected.

The larval stage is a translucent fluid filled bladder or cysticercus between $5-10 \mathrm{~mm}$ in diameter but, unlike T.solium cysticercus it has never been reliably described in humans.

The adult is large, white tapeworm that can reach 7-10 meters in length, weighing around 20-30 g. The scolex is 
equipped with suckers but not hooks. Mature proglottidis detach from the distal end of the worm and their independent emergence from anus is the principal cause of symptomatology.

Human infection is acquired by eating undercooked beef. Cattle are infected when their feed or grazing is contaminated by human feces ${ }^{4}$.

A majority of the patients are asymptomatic and do not have serious sequelae, However, sometimes they present with pruritus ani (77\%), nausea (46\%), abdominal pain $(43 \%)$, dizziness $(42 \%)$, increased appetite $(30 \%)$, and other mild gastrointestinal symptoms ${ }^{5}$.

Intestinal obstruction and perforation is commonly caused by Ascaris lumbricoides (Round worm). Other worms like Enterobius vermicularis (Pin worm), Trichuris trichura (Whip worm) and Taenia solium (Tape worm) can also rarely cause a similar picture ${ }^{6}$.

The incidence of the tapeworm infection has been reduced by proper preventive measures and antihelminthic drugs.

Praziquantel is the drug of first choice for T. saginata and $10-20 \mathrm{mg} / \mathrm{Kg}$ body weight should be given as a single oral dose ${ }^{7}$.

Niclosamide is the drug of second choice. T. saginata and T. solium should be differentiated from each other by microscopic examination of their mature segments and gravid proglottid and scolex ${ }^{8}$.

If parasitological diagnosis is uncertain, praziquantel is the preferred drug because of the danger of cysticercosis. The patient should be observed for several months as a new worm can regenerate if the scolex or a minute piece of neck remains viable.

We report this case as tape worm ( T. Saginata) being the rare cause of intestinal perforation, very few cases of perforation due to T.solium have been reported in literature ${ }^{9,10}$.

\section{Funding: Nil}

\section{Conflict of interest: Nil}

Permission from IRB: Yes

\section{References}

1. Sharma L, Gupta S, Soin AS, Sikora S, Kapoor V. Generalised peritonitis in India-The tropical spectrum. Jap J Surg 1991, 21:272-77.

2. Jhobta RS, Attri AK, Kaushik R, Sharma R, Jhobta A. Spectrum of perforation peritonitis in India-review of 504 consecutive cases. World J Emerg Surg. 2006; 1: 26

3. Abu-Salem1 OT, Hassan MA. Acute Abdomen and Taenia Solium Peritonei. Kuwait Medical Journal 2003, 35 (4): 299-300

4. Cook GC, Zumla AI . Manson's Tropical Diseases:. 22 $2^{\text {nd }}$ ed. UK: Saunders/ Elseiver ;2009. Other cestodes infections; p.1575-1577.

5. Hakeem SY, Rashid A, Suhail Khuroo S, Bali RS. Taenia saginata : A Rare Cause of Gall Bladder Perforation. Case Reports in Surgery. 2012(2012); id 572484, 3 pages.

6. Wani SA, Ahmad F, Zargar SA, Fomda BA, Ahmad Z, Ahmad P, "Helminthic infestation in children of Kupwara district: a prospective study. Indian Journal of Medical Microbiology. 2007;25(4) :398-400.

7. James WT, Leslie TW Jr. Goodman \& Gillman's Pharmacological Basis of Therapeutics, 10th ed. New York: McGraw Hill Co.; 2001. Antihelminthic Drugs; p.1123-1136.

8. Murray L, Ian BW. Oxford Handbook of Clinical Medicine, 6th ed. New York: Oxford University Press; 2006. Infectious Diseases: Cestodes (Tape worms); p.616617. 
9. Shrihari N, Mariraj J, Kumudini TS, Krishna S. Intestinal perforation due to tapeworm. Journal of clinical and Diagnostic Research. 2011;5(5): 1101-1103.
10. Faheem MK, Reddy KR, Nagaraja B, Rao SN, Reddy SE. Taenia induced ileal Perforation \& Peritonitis. J Bosci Tech. 2012;3(1):462-465.

\section{How to cite this article?}

Kurane SB, Kurane BT. Ileal Perforation Peritonitis Caused By T. Saginata: A Case Report. Int J Med Res Rev 2013;1(3):138141. doi:10.17511/ijmrr.2013.i03.11 